\title{
Incidence of Postoperative Pain after Single- and Multi-Visit Endodontic Treatment in Teeth with Vital and Non-Vital Pulp
}

\author{
Bayram Ince ${ }^{a}$ \\ Ertugrul Ercan ${ }^{b}$ \\ Mehmet Dallia \\ Coruh Turksel Dulgergil \\ Yahya Orcun Zorbac \\ Hakan Colak ${ }^{b}$
}

\section{ABSTRACT}

Objectives: To assess the incidence of postoperative pain after single- and multi-visit endodontic treatment of teeth with vital and non-vital pulp.

Methods: In total, 306 patients with teeth requiring endodontic treatment were identified and were included in this study. Two experienced clinicians treated the patients, who were randomly assigned to two groups. While the teeth of patients in group 1 were obturated, group 2 were temporarily sealed and obturated after one week. Three days after the root canal instrumentation of each tooth, the patients were asked whether they experienced any postoperative pain and to rate the level of discomfort as no, mild, moderate, or severe pain. Data were analyzed statistically using the chisquare test.

Results: No significant difference in postoperative pain was found between vital and non-vital teeth (P>.01). Mild, moderate, and severe pain occurred in 31.4, 13.7, and 4.6\% of vital teeth, respectively. Postoperative pain occurred in 107 (69.9\%) and 106 (69.3\%) teeth in the single- and multi-visit treatment groups, respectively. There was no significant difference in postoperative pain between the two groups $(P>$.01).

Conclusions: The prevalence of postoperative pain did not differ between vital and non-vital teeth. The majority of patients in either groups reported no or only mild pain. (Eur J Dent 2009;3:273-279)

Key words: Postoperative pain; Endodontic treatment; Non-vital teeth.

I a Department of Operative Dentistry, Faculty of Dentistry, Dicle University, Diyarbakir, Turkey.

b Department of Operative Dentistry, Faculty of Dentistry, University of Kirikkale, Kirikkale, Turkey.

Department of Endodontics, Faculty of Dentistry, University of Kirikkale, Kirikkale, Turkey.
Corresponding author:

Ertugrul Ercan

Department of Operative Dentistry, Faculty of

Dentistry, University of Kirikkale,

Kirikkale, Turkey.

E-mail: ertugrulercanahotmail.com 


\section{INTRODUCTION}

Pain is important in dentistry, because fear of pain is one of the major reasons for dental apprehension. Postoperative pain is one of the primary problems in endodontic treatment, even when proper anesthesia is provided. Although the success of endodontic treatment is highly related to the elimination or reduction of post-endodontic pain, many clinical studies have reported varying degrees of pain, ranging from 25 to $40 \%$. $^{1-5}$

Certain factors may influence the progression of postoperative pain, such as a history of preoperative pain and the need for re-treatment. ${ }^{6-8} \mathrm{Al}-$ though microorganisms are usually regarded as the most common cause of postoperative pain, other causes include mechanical or chemical injury to pulpal or periradicular tissues. ${ }^{9}$ There is a clear indication of interactions between periapical tissues and microorganisms, because flare-ups are more likely to occur in necrotic cases than in vital cases. ${ }^{10,11}$ This could indicate a clear relationship between pulp status and postoperative pain, even after successful endodontic therapy.3,12 However, limited data regarding the relationship between postoperative pain and the vitality of the pulp before endodontic treatment are available in the literature.

In irreversible pulpitis, ${ }^{13,14}$ endodontic therapy is currently the most frequently offered method to relieve pain. The completion of endodontic therapy in a single appointment has been currently used. ${ }^{3,15,16}$ Although the argument for single-visit treatment depends on the convenience, patient acceptance, and reduced postoperative pain, ${ }^{17}$ multiple-visit root canal therapy has long been taught to undergraduate dental students and is regarded as a safer procedure than single-visit root canal therapy. ${ }^{3,16}$

Current literature on single-visit versus multiple-visit endodontics ${ }^{18-20}$ provides conflicting opinions and recommendations, however, recent clinical reports, have shown that patients generally tolerate and prefer single-visit endodontic therapy. ${ }^{21-23}$ Therefore, single-visit root canal treatment has become a common practice and offers several advantages, including a reduced flare-up rate, decreased number of operative procedures, and no risk of inter-appointment leakage through temporary restorations. ${ }^{3,11,24}$ In addition, patient requests and expectations for treatment have made singlevisit root canal therapy popular among dental practitioners. Single-visit root canal therapy is less time consuming and more economical and, as a consequence, more appropriate to the needs of itinerant and busy patients. ${ }^{18,19,25,26}$

However, single-visit endodontic therapy has several disadvantages in terms of a good clinical experience and the potential for postoperative pain, even after successful root canal therapy. Furthermore, when flare-ups occur during multivisit procedures, they can be addressed before obturation, but this is not an option in a single-visit treatment regimen. ${ }^{27}$ In addition, bacterial eradication cannot be maximized predictably without using a calcium hydroxide dressing between appointments; therefore, the potential for healing may be compromised. ${ }^{28}$ This subject is very controversial, and opinions vary greatly as to the relative risks and benefits of single- versus multi-visit root canal treatment. ${ }^{29}$

Few studies have reported on the relationship between the number of treatment procedures and the occurrence of postoperative pain. 14,16,25 Therefore, in this clinical study, we determined the prevalence of pain after single- and multivisit endodontic treatment in vital versus non-vital teeth and evaluated the association between the prevalence of pain and pulp status.

\section{MATERIALS AND METHODS}

Three hundred and six patients who attended participating dental clinics for root canal treatment on one tooth only were invited to participate in this study. These patients, ranging in age from 18 to 60 years (average 45 years), were informed of the risks, aims, and possible conclusions of the study, and they signed informed consents.

All patients were in good health, as determined from a written health history and oral interview. Patients who had previously taken antibiotics or analgesics were excluded. Age, gender, tooth location, and the vitality of their teeth were recorded. An electric pulp-testing device (Parkell pulp vitality tester, Farmingdale, NY, USA) was used to assess pulp vitality.

Before initiating treatment, each tooth was scored according to clinical complaints, including the presence or absence of pain. Overall, 142 patients had symptomatic (preoperative pain) 
and 164 had asymptomatic teeth, respectively. Of the 153 teeth previously diagnosed as non-vital, 22 showed periapical lesions. Eighty-six of the asymptomatic teeth and 67 of the symptomatic teeth were treated in a single visit; the remaining patients (78 asymptomatic and 75 symptomatic teeth) were treated in multiple visits. Of the 22 teeth with lesions (about $3-5 \mathrm{~mm}$ ), 12 were treated in multiple visits.

Two trained clinicians (CTD, OZ) performed the clinical and radiographic examinations and determined which cases would be treated endodontically. A single clinician (CTD) re-evaluated all selected cases, using radiographic and clinical findings. This procedure was performed to eliminate or minimize interpersonal variability between clinicians. Furthermore, the same clinician was assigned for treatment of all cases selected for this study, and that clinician also randomly directed the cases to one of two operators (EE, MD) who would perform the clinical procedures. During this part of the study, patients were assigned consecutively to either single-visit or multiple-visit treatments by the same clinician, who re-evaluated all cases. Therefore, the case and operator distribution were blinded, and a separate blind clinician evaluated patient discomfort and pain between each visit (FY).

Two experienced clinicians carried out all clinical procedures. The standard procedure for both groups at the first appointment included local anesthesia with $1.8 \mathrm{~mL}$ of $4 \%$ prilocaine (prilocaine $\mathrm{HCl}$ injection $40 \mathrm{mg} / \mathrm{ml}$; Dentsply Pharmaceutical, York, PA, USA) by infiltration injection for maxillary teeth and by inferior alveolar nerve block injection for mandibular teeth, rubber dam isolation, caries excavation, and standard access preparation. The working length was determined radiographically from a coronal reference to a distance $1 \mathrm{~mm}$ short of the radiographic apex. The root canals were cleaned and shaped using the step-back technique, hand files, and Gates-Glidden drills (Dentsply/Maillefer, Ballaigues, Switzerland). Each file was followed by irrigation of the canal with 2 $\mathrm{mL}$ sodium hypochlorite $(5 \%)$ in a syringe with a 27-gauge needle. Irrigation was carried out with an endodontics Monoject syringe $(3 \mathrm{~mL}, 27$-gauge needle; Pierre Rolland, Mérignac, Francel to ensure that the irrigant approached the apex.

The teeth were then randomly assigned to two groups as follows: group 1, single-visit therapy (87 vital and 66 non-vital teeth); each root canal was dried with paper points, then filled with guttapercha points sealed with $\mathrm{AH}-26$ root canal sealer (Dentsply, Konstanz, Germany) using the lateral condensation technique. Group 2, multi-visit therapy (66 vital and 87 non-vital teeth); the teeth were prepared as in group 1, but were not obturated. Chemomechanical preparation was completed in the first visit using the same technique for all cases. A sterile cotton pellet was placed in the pulp chamber, and the access cavity was filled with quick-setting zinc oxide eugenol cement (Cavex, Haarlem, The Netherlands). One week later, the teeth were obturated as in group 1. The number of teeth that each of the clinicians treated in each experimental group were as follows: 79 and 74 in the single-visit group and 81 and 72 in the multi-visit group for operators A and B, respectively. For both groups, the number of teeth treated by the two different operators was not significantly different.

Although no systemic medication was prescribed, the patients were instructed to take mild analgesics (400 mg of ibuprofen [Brufen 400], Abbott, Turkeyl if they experienced pain. At the beginning of the second appointment, patients were asked about the occurrence of postoperative pain. The written instructions were followed by a phone call to the operators to establish if there were any difficulties in understanding or using the datacollection forms. The assessment of postoperative pain was carried out at 3 days after initial appointment by one independent evaluator without knowledge of visit group under examination. Three days after the initial appointment, the presence or absence of pain, or the appropriate degree of pain was recorded for each recall visit and the interval between visits. Pain was recorded as none, slight, moderate, or severe: ${ }^{16}$

- No pain: The treated tooth felt normal. Patients don't have any pain.

- Mild pain: Recognizable, but not discomforting, pain, which required no analgesics.

- Moderate pain: Discomforting, but bearable, pain lanalgesics, if used, were effective in relieving the pain).

- Severe pain: Difficult to bear lanalgesics had little or no effect in relieving the pain).

A follow-up evaluation was made of the radiographic and clinical data. During this follow-up 
period, the coronal restorations were found to be of good quality. The data were analyzed statistically using the chi-square test.

\section{RESULTS}

Table 1 details the distribution of the treated teeth according to tooth type in the two groups. The study comprised 306 patients (106 females and 200 males; Table 1). Of the 153 cases in the single-visit group, 48 were females and 105 were males. The difference between the number of male and female patients was significant $(P<.05)$.

The differences between the number of upper and lower teeth were not significant $(P>.05$; Table 1). The teeth were divided into mandibular and maxillary arches, resulting in 183 maxillary and 123 mandibular teeth that were included in the study (Table 1). Regarding the to tooth type, 64 incisors, 42 premolars, and 47 molars were treated in a single visit; the postoperative rates of severe and moderate pain for these single visits were $17(26.6 \%), 8$ (19\%), and 3 (6\%), respectively. Seventy incisors, 49 premolars, and 39 molars were treated in the multiple-visit group; the postoperative rates of severe and moderate pain were $13(18.6 \%), 6(12.2 \%)$, and 11 (6.4\%), respectively. The differences in rates among these groups were not significant $(P=0.088)$; however, with respect to tooth type, incisors tended to experience significantly greater postoperative pain.

When the incidence of pain was compared in the single- and multiple-visit groups (Table 2), the

Table 1. Distribution of various patient variables and the clinical properties of teeth in the complete healing group.

\begin{tabular}{l|c|c|c}
\hline Variables & Total (n) & Preoperative Pain (n) & Postoperative pain (n) \\
\hline Male & $200(65.4 \%)$ & $98(69.0 \%)$ & $120(56.3 \%)$ \\
Female & $106(34.6 \%)$ & $44(31.0 \%)$ & $93(43.7 \%)$ \\
\hline Age & & & \\
\hline $18-28$ & $115(37.6 \%)$ & $48(33.8 \%)$ & $69(32.4 \%)$ \\
$29-39$ & $83(27.1 \%)$ & $41(28.9 \%)$ & $67(31.5 \%)$ \\
\hline $40-50$ & $58(19.0 \%)$ & $21(14.8 \%)$ & $35(16.4 \%)$ \\
\hline $51-61$ & $29(9.5 \%)$ & $18(12.7 \%)$ & $21(9.9 \%)$ \\
\hline $61>$ & $21(6.9 \%)$ & $14(9.9 \%)$ & $21(9.9 \%)$ \\
\hline Tooth location & & & $64(30.0 \%)$ \\
Maxillary incisor/canine & $96(31.4 \%)$ & $34(23.9 \%)$ & $29(13.6 \%)$ \\
Maxillary premolars & $62(20.3 \%)$ & $22(15.5 \%)$ & $23(10.8 \%)$ \\
Maxillary molars & $25(8.2 \%)$ & $21(14.8 \%)$ & $54(25.4 \%)$ \\
Mandibular incisor/canine & $55(18.0 \%)$ & $39(27.5 \%)$ & $25(11.7 \%)$ \\
Mandibular premolar & $36(11.8 \%)$ & $17(12.0 \%)$ & $18(8.5 \%)$ \\
Mandibular molar & $32(10.5 \%)$ & $9(6.3 \%)$ & 11 \\
Presence of periapical lesion preoperatively & 22 & 9 & 212 \\
No periapical lesion preoperatively & 284 & 133 & \\
\hline
\end{tabular}

Table 2. Clinical symptoms and tooth types of vital and non-vital teeth in both treatment regimens.

\begin{tabular}{|c|c|c|c|c|c|c|c|c|c|c|}
\hline \multirow{3}{*}{ Type of treatment } & \multicolumn{6}{|c|}{ Tooth type } & \multicolumn{4}{|c|}{ Preoperative pain } \\
\hline & \multicolumn{2}{|c|}{ Incisors or canines } & \multicolumn{2}{|c|}{ Premolars } & \multicolumn{2}{|c|}{ Molars } & \multirow{2}{*}{ No } & \multirow{2}{*}{ Mild } & \multirow{2}{*}{ Moderate } & \multirow{2}{*}{ Severe } \\
\hline & $\mathrm{V}$ & DV & $\mathrm{v}$ & DV & V & DV & & & & \\
\hline Single visit & 48 & 33 & 26 & 23 & 13 & 10 & 86 & 37 & 18 & 12 \\
\hline Two visit & 19 & 51 & 24 & 25 & 23 & 11 & 78 & 44 & 12 & 19 \\
\hline Total & 67 & 84 & 50 & 48 & 36 & 21 & 164 & 81 & 30 & 31 \\
\hline
\end{tabular}


multiple-visit group reported significantly less pain than the single-visit group $(P<.01)$. A comparison of pain experience in relation to pre-treatment pulpal vitality between the 76 teeth with vital pulp and the 215 teeth with non-vital pulp (Table 3) revealed that those with vital pulp had a significantly lower frequency of pain (9\%) than those with non-vital pulp $(41 \% ; \mathrm{P}<.005)$.

Nineteen of the 87 patients with vital pulp in the single-visit group and 16 of the 66 patients with vital pulp in the multiple-visit group reported post-obturation pain. There was no significant difference in the pain experience between the two groups $(P<.05)$. In contrast, for patients with non-vital pulp, 14 of the 87 multiple-visit group reported post-obturation pain, while only nine of the 66 patients in the single-visit group reported pain. This difference was significant $(P<.005)$.

At the start of the study, we observed 22 cases with periapical lesions. Of those cases, 12 and 10 were treated in the single- and multi-visit treatment groups, respectively. Of these, nine had preoperative pain and 11 had postoperative pain. For teeth with previous periapical lesions, the association between the presence of preoperative pain and the presence of postoperative pain was not statistically significant ( $P>$.05).

The distribution of preoperative pain has been summarized in Table 2 according to the different degrees of pain. For both groups, the distribution of postoperative pain is presented in Tables 3 and 4. Postoperative pain was reported by 107 (69.9\%) and 106 (69.3\%) patients in the single- and multi-visit treatment groups, respectively. No significant difference in postoperative pain was found between the two groups ( $P>.01)$, and pain did not occur in $46(30.1 \%)$ and $47(30.7 \%)$ patients in the single- and multi-visit groups, respectively. However, severe pain was reported by $7(4.6 \%)$ and $11(7.2 \%)$ patients, respectively, from the singleand multi-visit groups; and mild pain occurred in $51.6 \%$, moderate pain in $13.7 \%$, and severe pain (flare-up) in $4.6 \%$ of all cases $(79,21$, and 7 patients, respectively; Table 4).

\section{DISCUSSION}

This study clearly showed that the degree and frequency of postoperative pain related to singleand multi-visit endodontic treatment did not differ between the two groups. Consequently, one-visit therapy had some advantages, including reducing the number of operative procedures.

Often, clinicians had patients with postoperative pain, even after successful endodontic therapy. To eliminate these inter-individual differences, in this study, we randomly selected patients with only one tooth for treatment, as multiple teeth in the same patient could not be assumed to be-

Table 3. Distribution of pain level and tooth type in the single-visit group for both vital and non-vital teeth.

\begin{tabular}{lccccccccc}
\hline & \multicolumn{9}{c}{ Single-visit } \\
Tooth groups & \multicolumn{2}{c}{ No pain } & \multicolumn{2}{c}{ Mild pain } & Moderate pain & \multicolumn{2}{c}{ Severe pain } \\
& Vital & Nonvital & Vital & Nonvital & Vital & Nonvital & Vital & Nonvital \\
\hline Incisors or canines & 11 & 6 & 25 & 5 & 9 & 4 & 3 & 1 \\
Premolars & 11 & 3 & 10 & 10 & 3 & 2 & 2 & 1 \\
Molars & 5 & 10 & 6 & 23 & 2 & 1 & 0 & 0 \\
Total & 27 & 19 & 41 & 28 & 14 & 7 & 5 & 2 \\
\hline
\end{tabular}

Table 4. Distribution of pain level and tooth type in the multi-visit group for both vital and non-vital teeth.

\begin{tabular}{lccccccccc}
\hline & \multicolumn{9}{c}{ Multi-visit } \\
Tooth groups & \multicolumn{2}{c}{ No pain } & \multicolumn{2}{c}{ Mild pain } & \multicolumn{2}{c}{ Moderate pain } & \multicolumn{2}{c}{ Severe pain } \\
& Vital & Nonvital & Vital & Nonvital & Vital & Nonvital & Vital & Nonvital \\
\hline Incisors or canines & 5 & 11 & 8 & 33 & 4 & 5 & 2 & 2 \\
Premolars & 12 & 11 & 10 & 10 & 0 & 2 & 2 & 2 \\
Molars & 5 & 3 & 10 & 5 & 7 & 1 & 1 & 2 \\
Total & 22 & 25 & 28 & 48 & 11 & 8 & 5 & 6 \\
\hline
\end{tabular}


have independently. This fact agrees with previous studies of postoperative pain levels. ${ }^{30}$

A number of factors concerning the etiology of postoperative pain have been evaluated. For example, a clear indication of the relationship between microbial interactions and periapical tissues is that flare-ups are more likely to occur in necrotic cases (infected) than in vital cases (non-infected). ${ }^{10,11}$ We found no significant difference in postoperative pain between vital and nonvital teeth $(P>$.01). Our results disagree with some previous studies that reported an increased incidence of postoperative pain or flare-ups in cases with necrotic pulp. ${ }^{3,31,32}$ However, our results were similar to Genet et $\mathrm{al}^{12}$ and Yesilsoy et $\mathrm{al}^{33}$ showing that postoperative pain was not related to the pulp condition.

A number of factors concerning the etiology of postoperative pain have been evaluated. For example, a clear indication of the relationship between microbial interactions and periapical tissues is that flare-ups are more likely to occur in necrotic cases (infected) than in vital cases (noninfected). ${ }^{10,11}$ We found no significant difference in postoperative pain between vital and non-vital teeth $(P>$.01). Our results disagree with some previous studies ${ }^{3,31,32}$ that reported an increased incidence of postoperative pain or flare-ups in cases with necrotic pulp. However, our results were similar to those of other investigations ${ }^{12,33}$ showing that postoperative pain was not related to the condition of the pulp.

In addition to various factors, such as tooth type and location, the presence and severity of preoperative pain, pulpal status, and the presence and size of a periapical lesion, the number of treatment visits also has a significant effect on postoperative pain due to the high risk of inter-appointment microbial leakage through temporary restorations. ${ }^{34,35}$

In retrospective studies, Smith et $\mathrm{al}^{36}$ and Jenkins et $a^{37}$ determined that there was a $5.5 \%$ incidence of pain (flare-ups) in patients who had pulp necrosis and asymptomatic periapical lesions. This finding was supported by a study in which only two of 22 teeth with periapical pathosis developed postoperative pain after single-visit endodontic treatment. ${ }^{35}$

\section{CONCLUSIONS}

Our results clearly indicate that postoperative pain after successful endodontic therapy is mainly related to preoperative pain rather than the clinical or radiographic diagnosis; they also show that there were no differences between single- and multi-visit treatment protocols and vital and nonvital teeth with respect to the incidence of postoperative pain.

\section{REFERENCES}

1. Holstein A, Hargreaves KM, Neiderman R. Evaluation of NSAIDs for treating post-endodontic pain. Endod Topics 2002;3:3-13.

2. Torabinejad M, Cymerman JJ, Frankson M, Lemon RR, Maggio JD, Schilder H. Effectiveness of various medications on postoperative pain following complete instrumentation. $J$ Endod 1994;20:345-354.

3. Albashaireh ZS, Alnegrish AS. Postobturation pain after single and multiple-visit endodontic therapy. $J$ Dent 1998;26:227-232.

4. Rogers MJ, Johnson BR, Remeikis NA, BeGole EA. Comparison of effect of intracanal use of ketorolac tromethamine and dexamethasone with oral ibuprofen on post treatment endodontic pain. J Endod 1999;25:381-384.

5. DiRenzo A, Gresla T, Johnson BR, Rogers M, Tucker D, BeGole EA. Postoperative pain after 1- and 2-visit root canal therapy. Oral Surg Oral Med Oral Pathol Oral Radiol Endod 2002;93:605-610

6. Torabinejad M, Kettering JD, McGraw JC, Cummings RR, Dwyer TG, Tobias TS. Factors associated with endodontic interappointment emergencies of teeth with necrotic pulps. J Endod 1988;14:261-266.

7. Imura N, Zuolo ML. Factors associated with endodontic flare-ups: a prospective study. Int Endod J 1995;28:261265.

8. Morse DR, Koren LZ, Esposito JV, Goldberg JM, Sinai IH, Furst ML. Asymptomatic teeth with necrotic pulps and associated periapical radiolucencies: relationship of flareups to endodontic instrumentation, antibiotic usage and stress in three separate practices at three different time periods. Parts 1-5. Int J Psychosom 1986;33:5-87.

9. Siqueira JF Jr, Rocas IN, Favieri A, Machado AG, Gahyva SM, Oliveira JC, Abad EC. Incidence of postoperative pain after intracanal procedures based on an antimicrobial strategy. J Endod 2002;28:457-460.

10. Seltzer S, Naidorf IJ. Flare-ups in endodontics: I. Etiological factors. J Endod 1985;11:472-478. 
11. Walton R, Fouad A. Endodontic interappointment flareups: a prospective study of incidence and related factors. $J$ Endod 1992;18:172-177.

12. Genet JM, Wesselink PR, Thoden Van Velzen SK. Preoperative and operative factors associated with pain after the first endodontic visit. Int Endod $J$ 1987;20:53-64.

13. Rahbaran S, Gilthorpe MS, Harrison SV, Gulabivala K. Comparison of clinical outcome of periapical surgery in Endodontic and Oral Surgery units of a teaching Dental Hospital: a retrospective study. Oral Surg Oral Med Oral Pathol Oral Radiol Endod 2001;91:700-709.

14. Eleazer PD, Eleazer KR. Flare-up rate in pulpally necrotic molars in one-visit versus two-visit endodontic treatment. J Endod 1998;24:614-616.

15. Qualtrough AJ, Whitworth JM, Dummer PM: Preclinical endodontology: an international comparison. Int Endod $J$ 1999,32:406-414.

16. Oginni A, Udoye C. Endodontic flare-ups: comparison of incidence between single and multiple visit procedures in patients attending a Nigerian teaching hospital. BMC Oral Health 2004,4:4-6.

17. Inamoto K, Kojima K, Nagamatsu K, Hamaguchi A, Nakata $\mathrm{K}$, Nakamura H. A survey of the incidence of single-visit endodontics. J Endod 2002;28:371-374.

18. Pekruhn RB. Single-visit endodontic therapy: a preliminary clinical study. J Am Dent Assoc 1981;103:875-877.

19. Jurcak JJ, Bellizzi R, Loushine RJ. Successful singlevisit endodontics during Operation Desert Shield. J Endod 1993;198:412-413.

20. Welch I. One appointment endodontic treatment. J Can Dent Assoc 1975;41:613-615.

21. Fava LR. A comparison of one versus two appointment endodontic therapy in teeth with non-vital pulps. Int Endod $J$ 1989;22:179-183.

22. Oliet S. Single-visit endodontics: a clinical study. J Endod 1983;9:147-152.

23. Roane JB, Dryden JA, Grimes EW. Incidence of postoperative pain after single- and multiple-visit endodontic procedures. Oral Surg Oral Med Oral Pathol 1983;55:68-72.

24. Cohen S, Burns RC. Pathways of the Pulp. $7^{\text {th }}$ ed. St. Louis: CV Mosby, 1998:73-96.

25. Field JW, Gutmann JL, Solomon ES, Rakusin H. A clinical radiographic retrospective assessment of the success rate of single-visit root canal treatment. Int Endod J 2004;37:7082.

26. Weiselberg M, Vogelson K. One-visit endodontics: not only could you but should you? J NJ Dent Assoc 1983;54:59-63.

27. Soltanoff W. A comparative study of the single-visit and the multiple-visit endodontic procedure. J Endod 1978;11:278281.
28. Spångberg LS. Evidence-based endodontics: the one-visit treatment idea [Comment]. Oral Surg Oral Med Oral Pathol Oral Radiol Endod 2001;91:617-618.

29. Sathorn C, Parashos P, Messer HH. Effectiveness of single-versus multiple-visit endodontic treatment of teeth with apical periodontitis: a systematic review and metaanalysis. Int Endod $J$ 2005;38:347-355.

30. Alaçam T, Tınaz AC. Interappointment emergencies in teeth with necrotic pulps. J Endod 2002;28:375-377.

31. Clem WH. Posttreatment endodontic pain. J Am Dent Assoc 1970;81:1166-1670.

32. Mor C, Rotstein I, Friedman S. Incidence of interappointment emergency associated with endodontic therapy. $J E n-$ dod 1992;18:509-511.

33. Yesilsoy C, Korzen LZ, Morse DR, Rankow H, Bolanos OR, Furst ML. Post-endodontic obturation pain: a comparative evaluation. Quintessence Int 1988;19:431-438.

34. Harrison JW, Baumgartner JC, Svec TA. Incidence of pain associated with clinical factors during and after root canal therapy. Part 2. Postobturation pain. J Endod 1983;9:434438.

35. Fox J, Atkinson JS, Dinin AP, Greenfield E, Hechtman E, Reeman CA, Salkind M, Todaro CJ. Incidence of pain following one-visit endodontic treatment. Oral Surg Oral Med Oral Pathol 1970;30:123-130.

36. Smith CS, Setchell DJ, Harty FJ. Factors influencing the success of conventional root canal therapy - a 5-year retrospective study. Int Endod $J$ 1993;26:321-333.

37. Jenkins SM, Hayes SJ, Dummer PMH. A study of endodontic treatment carried out in dental practice within the UK. Int Endod J 2001;34;16-22. 